\title{
Schwarz lemma involving the boundary fixed point
}

Qinghua $\mathrm{Xu}^{1{ }^{*},}$, Yongfa Tang ${ }^{1}$, Ting Yang ${ }^{1}$ and Hari Mohan Srivastava ${ }^{2,3}$

\section{"Correspondence:}

xugh@mail.ustc.edu.cn

${ }^{1}$ College of Mathematics and Information Science, JiangXi Normal University, NanChang, 330022, People's Republic of China

Full list of author information is available at the end of the article

\begin{abstract}
Let $f$ be an holomorphic function which maps the unit disk into itself. In this paper, consider the zero of order $k(i . e ., f(z)-f(0)($ or $f(z))$ has a zero of order $k$ at $z=0)$, we obtain the sharp estimates of the classical boundary Schwarz lemma involving the boundary fixed point. The results presented here would generalize the corresponding result obtained by Frolova et al. (Complex Anal. Oper. Theory 8:1129-1149, 2004).
\end{abstract}

MSC: $30 C 45 ; 32 \mathrm{~A} 10$

Keywords: boundary Schwarz lemma; fixed point; zero of order

\section{Introduction and preliminaries}

It is well known that the Schwarz lemma serves as a very powerful tool to study several research fields in complex analysis. For example, almost all results in the geometric function theory have the Schwarz lemma lurking in the background [2-6].

On the other hand, Schwarz lemma at the boundary is also an active topic in complex analysis, various interesting results have been obtained [7-14]. Before summarizing these results, it is necessary to give some elementary contents on the boundary fixed points [15].

Let $\mathbb{D}$ denote the unit disk in $\mathbb{C}, H(\mathbb{D}, \mathbb{D})$ denote the class of holomorphic self-mappings of $\mathbb{D}, \mathbb{N}$ denote the set of all positive integers. The boundary point $\xi \in \partial \mathbb{D}$ is called a fixed point of $f \in H(\mathbb{D}, \mathbb{D})$ if

$$
f(\xi)=\lim _{r \rightarrow 1^{-}} f(r \xi)=\xi
$$

The classification of the boundary fixed points of $f \in H(\mathbb{D}, \mathbb{D})$ can be performed via the value of the angular derivative

$$
f^{\prime}(\xi)=\angle \lim _{z \rightarrow \xi} \frac{f(z)-\xi}{z-\xi}
$$

which belongs to $(0, \infty]$ due to the celebrated Julia-Carathédory theorem [13]. This theorem also asserts that the finite angular derivative at the boundary fixed point $\xi$ exists if and only if the holomorphic function $f^{\prime}(z)$ has the finite angular $\operatorname{limit} \angle \lim _{z \rightarrow \xi} f^{\prime}(z)$. For a boundary fixed point $\xi$ of $f$, if

$$
f^{\prime}(\xi) \in(0, \infty)
$$

(c) $2016 \mathrm{Xu}$ et al. This article is distributed under the terms of the Creative Commons Attribution 4.0 International License (http://creativecommons.org/licenses/by/4.0/), which permits unrestricted use, distribution, and reproduction in any medium, provided you give appropriate credit to the original author(s) and the source, provide a link to the Creative Commons license, and indicate if changes were made. 
then $\xi$ is called a regular boundary fixed point. The regular fixed points can be attractive if $f^{\prime}(\xi) \in(0,1)$, neutral if $f^{\prime}(\xi)=1$, or repulsive if $f^{\prime}(\xi) \in(1, \infty)$.

By the Julia-Carathédory theorem [13] (see also [7]) and the Wolff lemma [11], if $f \in$ $H(\mathbb{D}, \mathbb{D})$ with no interior fixed point, then there exists a unique regular boundary fixed point $\xi$ such that $f^{\prime}(\xi) \in(0,1]$; and if $f \in H(\mathbb{D}, \mathbb{D})$ with an interior fixed point, then $f^{\prime}(\xi)>1$ for any boundary fixed point $\xi \in \partial \mathbb{D}$.

In particular, Unkelbach [16] (see also [17]) obtain the following boundary Schwarz lemma.

Theorem A Iff $\in H(\mathbb{D}, \mathbb{D})$ has a regular boundary fixed point 1 , and $f(0)=0$, then

$$
f^{\prime}(1) \geq \frac{2}{1+\left|f^{\prime}(0)\right|} \text {. }
$$

Moreover, equality in (1) holds if and only iff is of the form

$$
f(z)=-z \frac{a-z}{1-a z}, \quad \forall z \in \mathbb{D},
$$

for some constant $a \in(-1,0]$.

Theorem A was improved 60 years later by Osserman [18] by removing the assumption $f(0)=0$.

Theorem $\mathbf{B}([18])$ Iff $\in H(\mathbb{D}, \mathbb{D})$ with $\xi=1$ as its regular boundary fixed point. Then

$$
f^{\prime}(1) \geq \frac{2(1-|f(0)|)^{2}}{1-|f(0)|^{2}+\left|f^{\prime}(0)\right|}
$$

In [1], Frolova et al. proved the following theorem, which is an improvement of Theorem $B$.

Theorem $\mathbf{C}([1])$ If $\in H(\mathbb{D}, \mathbb{D})$ with $\xi=1$ as its regular boundary fixed point. Then

$$
f^{\prime}(1) \geq \frac{2}{\Re e\left(\frac{1-f(0)^{2}+f^{\prime}(0)}{(1-f(0))^{2}}\right)} .
$$

Recently, Ren and Wang [15] offered an alternative and elementary proof of Theorem C and studied the extremal functions of the inequality (3). Their method of proof is quite different from that which Frolova et al. have used in [1].

In this paper, stimulated by the above-cited work (especially [15]), considering the zero of order, we obtain a version of boundary Schwarz lemma. This result is a generalization of the boundary Schwarz-Pick lemma obtained by Frolova et al. [1].

In order to prove the desired results, we first recall the classical Julia lemma [3] and the Julia-Carathéodory theorem [19].

Lemma 1 ([3]) Let $f \in H(\mathbb{D}, \mathbb{D})$ and let $\xi \in \partial \mathbb{D}$. Suppose that there exists a sequence $\left\{z_{n}\right\}_{n \in \mathbb{N}} \subset \mathbb{D}$ converging to $\xi$ as $n$ tends to $\infty$, such that the limits

$$
\alpha=\lim _{n \rightarrow \infty} \frac{1-\left|f\left(z_{n}\right)\right|}{1-\left|z_{n}\right|}
$$


and

$$
\eta=\lim _{n \rightarrow \infty} f\left(z_{n}\right)
$$

exist (finitely). Then $\alpha>0$ and the inequality

$$
\frac{|f(z)-\eta|^{2}}{1-|f(z)|^{2}} \leq \alpha \frac{|z-\xi|^{2}}{1-|z|^{2}}
$$

holds throughout the open unit disk $\mathbb{D}$ and is strict except for Möbius transformations of $\mathbb{D}$.

Lemma $2([19])$ Let $f \in H(\mathbb{D}, \mathbb{D})$ and let $\xi \in \partial \mathbb{D}$. Then the following conditions are equivalent:

(i) The lower limit

$$
\alpha=\liminf _{z \rightarrow \xi} \frac{1-|f(z)|}{1-|z|}
$$

is finite, where the limit is taken as $z$ approaches $\xi$ unrestrictedly in $\mathbb{D}$;

(ii) $f$ has a non-tangential limit, say $f(\xi)$, at the point $\xi$, and the difference quotient

$$
\frac{f(z)-f(\xi)}{z-\xi}
$$

has a non-tangential limit, say $f(\xi)$, at the point $\xi$;

(iii) the derivative $f^{\prime}$ has a non-tangential limit, say $f^{\prime}(\xi)$, at the point $\xi$. Moreover, under the above conditions we have:

(a) $\alpha$ in (i);

(b) the derivatives $f^{\prime}(\xi)$ in (ii) and (iii) are the same;

(c) $f^{\prime}(\xi)=\alpha \bar{\xi} f(\xi)$;

(d) the quotient $\frac{1-|f(z)|}{1-|z|}$ has the non-tangential limit $\alpha$, at the point $\xi$.

Lemma $3\left([17]\right.$, p.35) Let $\varphi \in H(\mathbb{D}, \mathbb{D})$, and $\varphi(z)=\sum_{n=0}^{\infty} b_{n} z^{v}$. Then

$$
\left|b_{n}\right| \leq 1-\left|b_{0}\right|^{2}, \quad n \geq 1
$$

\section{Main results and their proofs}

We now state and prove each of our main results given by Theorems 1 and 2 below.

Theorem 1 Let $f \in H(\mathbb{D}, \mathbb{D})$ with $\xi=1$ as its regular boundary fixed point and suppose $f(0)=f^{\prime}(0)=\cdots=f^{(k-1)}(0)=0, a_{k}=\frac{f^{(k)}(0)}{k !} \neq 0, k \in \mathbb{N}$, we can obtain:

(I) if $0<\left|a_{k}\right|<1$, then

$$
f^{\prime}(1) \geq k+\frac{\left|1-a_{k}\right|^{2}}{1-\left|a_{k}\right|^{2}} \frac{2}{1+\Re \mathrm{e} \frac{1-\overline{a_{k}}}{1-a_{k}} \frac{a_{k+1}}{1-\left|a_{k}\right|^{2}}}
$$


where $a_{k+1}=\frac{f^{(k+1)}(0)}{(k+1) !}$. Equality holds in the inequality if and only iff is of the form

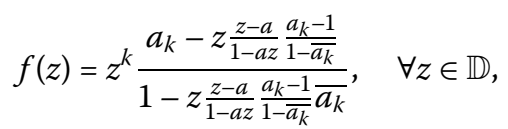

for some constant $a \in[-1,1)$.

(II) If $\left|a_{k}\right|=1$, then $f(z)=z^{k}$.

Proof In view of Lemma 3, we consider the following two cases.

Case I If $0<\left|a_{k}\right|<1$, let

$$
g(z)= \begin{cases}\frac{1-\overline{a_{k}}}{a_{k}-1} \frac{a_{k}-\frac{f(z)}{z^{k}}}{1-\overline{a_{k}} \frac{f(z)}{z^{k}}}, & 0<|z|<1, \\ 0, & z=0 .\end{cases}
$$

It is elementary to see that $g \in H(\mathbb{D}, \mathbb{D})$, and $\xi=1$ is its regular boundary fixed point. A straightforward computation shows that

$$
f^{\prime}(1)=k+\frac{\left|1-a_{k}\right|^{2}}{1-\left|a_{k}\right|^{2}} g^{\prime}(1)
$$

and

$$
g^{\prime}(0)=\frac{1-\overline{a_{k}}}{1-a_{k}} \cdot \frac{a_{k+1}}{1-\left|a_{k}\right|^{2}}
$$

which is no larger than 1 in modulus. Applying Lemmas 1 and 2 to the holomorphic function $h: \mathbb{D} \rightarrow \overline{\mathbb{D}}$ defined by

$$
h(z)=\frac{g(z)}{z}, \quad \forall z \in \mathbb{D}
$$

we obtain

$$
g^{\prime}(1)=1+h^{\prime}(1) \geq 1+\frac{\left|1-g^{\prime}(0)\right|^{2}}{1-\left|g^{\prime}(0)\right|^{2}}=\frac{2\left(1-\Re \operatorname{ee} g^{\prime}(0)\right)}{1-\left|g^{\prime}(0)\right|^{2}} .
$$

In particular,

$$
g^{\prime}(1) \geq \frac{2}{1+\Re \operatorname{Re} g^{\prime}(0)} .
$$

By combining (8), (9), and (11), we get the estimate in (6).

Furthermore, this bound in (6) is sharp. Indeed, if equality holds in (6) for $z \in \mathbb{D}$, then we must have equalities in the corresponding inequalities in (4) and (11). Thus, we can obtain

$$
g(z)=z \frac{z-a}{1-\bar{a} z} \frac{1-\bar{a}}{1-a}
$$

for some constant $a \in \overline{\mathbb{D}}$, and $g^{\prime}(0) \in(-1,1]$, which is possible only if $a \in[-1,1)$. 
Consequently, $f$ must be of the form

$$
f(z)=z^{k} \frac{a_{k}-z \frac{z-a}{1-a z} \frac{a_{k}-1}{1-\overline{a_{k}}}}{1-z \frac{z-a}{1-a z} \frac{a_{k}-1}{1-\bar{a}_{k}} \overline{\overline{a_{k}}}}, \quad \forall z \in \mathbb{D},
$$

for some constant $a \in[-1,1)$.

Case II If $\left|a_{k}\right|=1$, set

$$
g(z)= \begin{cases}\frac{f(z)}{z^{k}}, & 0<|z|<1, \\ a_{k}, & z=0 .\end{cases}
$$

It is clear that $g \in H(\mathbb{D}, \mathbb{D}),|g(0)|=\left|a_{k}\right|=1$. Thus by the principle of the maximum modulus, $g$ is a constant function, and $g(z)=a_{k}=g(1)=1$, and hence $f(z) \equiv z^{k}$. This completes the proof.

Taking into account the relation $\left|\frac{1-\overline{a_{k}}}{1-a_{k}} \cdot \frac{a_{k+1}}{1-\left|a_{k}\right|^{2}}\right| \leq 1$ and using (6) in Theorem 1, we can readily deduce the following corollary (the proof is omitted here).

Corollary 1 Let $f \in H(\mathbb{D}, \mathbb{D})$ with $\xi=1$ as its regular boundary fixed point and suppose $f(0)=f^{\prime}(0)=\cdots=f^{(k-1)}(0)=0, a_{k}=\frac{f^{(k)}(0)}{k !} \neq 0, k \in \mathbb{N}$; we have the following.

If $0<\left|a_{k}\right|<1$, then

$$
f^{\prime}(1) \geq k+\frac{\left|1-a_{k}\right|^{2}}{1-\left|a_{k}\right|^{2}}
$$

In particular,

$$
f^{\prime}(1) \geq k-1+\frac{2}{1+\Re \mathrm{e} a_{k}} .
$$

Remark 1 When $n=1$, it follows from (15) that

$$
f^{\prime}(1) \geq \frac{2}{1+\Re \mathrm{e} a_{1}}=\frac{2}{1+\Re \mathrm{e} f^{\prime}(0)}
$$

Note that

$$
\frac{2}{1+\Re f^{\prime}(0)} \geq \frac{2}{1+\left|f^{\prime}(0)\right|}
$$

Therefore, Theorem 1 (or Corollary 1) generalizes and improves Theorem A.

Theorem 2 Let $f \in H(\mathbb{D}, \mathbb{D})$ with $\xi=1$ as its regular boundary fixed point and suppose $f^{\prime}(0)=\cdots=f^{(k-1)}(0)=0, a_{k}=\frac{f^{(k)}(0)}{k !} \neq 0, k \in \mathbb{N}$, we can obtain:

(I) If $0<\left|a_{k}\right|<1-|f(0)|^{2}$, then

$$
f^{\prime}(1) \geq(k-1) \frac{|1-f(0)|^{2}}{1-|f(0)|^{2}}+\frac{2}{\Re \mathrm{e}\left(\frac{1-f^{2}(0)+a_{k}}{(1-f(0))^{2}}\right)} .
$$


Equality holds in the inequality if and only if $f$ is of the form

$$
f(z)=\frac{f(0)-z^{k} \frac{a-z}{1-a z} \frac{1-f(0)}{1-\overline{f(0)}}}{1-z^{k} \frac{a-z}{1-a z} \frac{1-f(0)}{1-\overline{f(0)}} \overline{f(0)}}
$$

(II) If $\left|a_{k}\right|=1-|f(0)|^{2}$, then

$$
f(z)=\frac{\frac{1-f(0)}{1-\overline{f(0)}} z^{k}+f(0)}{1+\overline{f(0)} \frac{1-f(0)}{1-\overline{f(0)}} z^{k}}
$$

Proof Set

$$
g(z)=\frac{f(z)-f(0)}{1-\overline{f(0)} f(z)} \frac{1-\overline{f(0)}}{1-f(0)}
$$

It is not difficult to verify that $g \in H(\mathbb{D}, \mathbb{D})$, and $\xi=1$ is its regular boundary fixed point. Elementary computations yield

$$
f^{\prime}(1)=\frac{|1-f(0)|^{2}}{1-|f(0)|^{2}} g^{\prime}(1)
$$

and

$$
\frac{g^{(k)}(0)}{k !}=\frac{a_{k}}{1-|f(0)|^{2}} \frac{1-\overline{f(0)}}{1-f(0)}
$$

On the other hand, let

$$
h(z)= \begin{cases}\frac{g(z)}{z^{k}}, & 0<|z|<1 \\ \frac{g^{(k)}(0)}{k !}, & z=0\end{cases}
$$

which is in $H(\mathbb{D}, \mathbb{D})$. By Lemma 3, we obtain the following results:

(I) If $0<\left|a_{k}\right|<1-|f(0)|^{2}$, then it follows from (19) that $\left|\frac{g^{(k)}(0)}{k !}\right|<1$. By using Lemmas 1 and 2 , we have

$$
g^{\prime}(1)=k+h^{\prime}(1) \geq k+\frac{\left|1-\frac{g^{(k)}(0)}{k !}\right|^{2}}{1-\left|\frac{g^{(k)}(0)}{k !}\right|^{2}}=k-1+\frac{2\left(1-\Re \mathrm{e} \frac{g^{(k)}(0)}{k !}\right)}{1-\left|\frac{g^{(k)}(0)}{k !}\right|^{2}} .
$$

In particular,

$$
g^{\prime}(1) \geq k-1+\frac{2}{1+\mathfrak{R e} \frac{g^{(k)}(0)}{k !}} .
$$

From the above relation and (18), we deduce that

$$
f^{\prime}(1) \geq \frac{|1-f(0)|^{2}}{1-|f(0)|^{2}}\left(k-1+\frac{2}{1+\Re \mathrm{e} \frac{g^{(k)}(0)}{k !}}\right)
$$




$$
\begin{aligned}
& =\frac{|1-f(0)|^{2}}{1-|f(0)|^{2}}\left(k-1+\frac{2}{1+\Re \mathrm{e}\left(\frac{a_{k}}{1-|f(0)|^{2}} \frac{1-\overline{f(0)}}{1-f(0)}\right)}\right) \\
& =(k-1) \frac{|1-f(0)|^{2}}{1-|f(0)|^{2}}+\frac{2}{\Re \mathrm{e}\left(\frac{1-f^{2}(0)+a_{k}}{(1-f(0))^{2}}\right)} .
\end{aligned}
$$

Applying a similar argument to Theorem 1, we deduce that equality holds in inequality (16) if and only if $f$ is of the form

$$
f(z)=\frac{f(0)-z^{k} \frac{a-z}{1-a z} \frac{1-f(0)}{1-\overline{f(0)}}}{1-z^{k} \frac{a-z}{1-a z} \frac{1-f(0)}{1-\overline{f(0)}} \overline{f(0)}} .
$$

(II) If $\left|a_{k}\right|=1-|f(0)|^{2}$, then we find from (19) and (20) that $|h(0)|=\left|\frac{g^{(k)}(0)}{k !}\right|=1$. By the principle of the maximum modulus, $h$ is a constant function, and $h(z)=g(1)=1$, and hence $g(z) \equiv z^{k}$, which yields the assertion (17). This completes the proof.

Remark 2 By setting $k=1$ in (16) of Theorem 2, we get the following estimate:

$$
f^{\prime}(1) \geq \frac{2}{\Re e\left(\frac{1-f^{2}(0)+a_{1}}{(1-f(0))^{2}}\right)}=\frac{2}{\Re e\left(\frac{1-f(0)^{2}+f^{\prime}(0)}{(1-f(0))^{2}}\right)}
$$

which is Theorem C obtained by Frolova et al. [1]. Thus, Theorem 2 is a generalization of Theorem C.

Competing interests

The authors declare that they have no competing interests.

Authors' contributions

The authors contributed equally to this work.

\section{Author details}

${ }^{1}$ College of Mathematics and Information Science, JiangXi Normal University, NanChang, 330022, People's Republic of China. ${ }^{2}$ Department of Mathematics and Statistics, University of Victoria, Victoria, British Columbia V8W 3P4, Canada.

${ }^{3}$ China Medical University, Taichung, 40402, Taiwan, People's Republic of China.

\section{Acknowledgements}

This work was supported by NNSF of China (Grant Nos. 11561030, 11261022), the Jiangxi Provincial Natural Science Foundation of China (Grant No. 20152ACB20002), and Natural Science Foundation of Department of Education of Jiangxi Province, China (Grant No. GJJ150301)

Received: 16 March 2016 Accepted: 4 August 2016 Published online: 16 August 2016

\section{References}

1. Frolova, A, Levenshtein, M, Shoikhet, D, Vasil'ev, A: Boundary distortion estimates for holomorphic maps. Complex Anal. Oper. Theory 8, 1129-1149 (2004)

2. Abate, M: Iteration Theory of Holomorphic Maps on Taut Manifolds. Mediterranean Press, Rende (1989)

3. Elin, M, Jacobzon, F, Levenshtein, M, Shoikhet, D: The Schwarz lemma: rigidity and dynamics. In: Harmonic and Complex Analysis and Its Applications, pp. 135-230. Springer, Cham (2014)

4. Dai, SY, Chen, HH, Pan, YF: The Schwarz-Pick lemma of high order in several variables. Mich. Math. J. 59(3), 517-533 (2010)

5. Ruscheweyh, S: Two remarks on bounded analytic functions. Serdica 11, 200-202 (1985)

6. Liu, Y, Chen, ZH: Schwarz-Pick estimates for holomorphic mappings from the polydisk to the unit ball. J. Math. Anal. Appl. 376, 123-125 (2011)

7. Anderson, JM, Vasil'ev, A: Lower Schwarz-Pick estimates and angular derivatives. Ann. Acad. Sci. Fenn., Math. 33 $101-110(2008)$

8. Cowen, CC, Pommerenke, C: Inequalities for the angular derivative of an analytic function in the unit disk. J. Lond. Math. Soc. 26(2), 271-289 (1982)

9. Bolotnikov, V, Elin, M, Shoikhet, D: Inequalities for angular derivatives and boundary interpolation. Anal. Math. Phys. 3(1), 63-96 (2013) 
10. Elin, M, Shoikhet, D, Tarkhanov, N: Separation of boundary singularities for holomorphic generators. Ann. Mat. Pura Appl. 190, 595-618 (2011)

11. Wolff, J: Sur une generalisation d'un theoreme de Schwarz. C. R. Acad. Sci. 182, 918-920 (1926)

12. Julia, G: Extension nouvelle d'un lemme de Schwarz. Acta Math. 42, 349-355 (1920)

13. Carathéodory, C: Uber die Winkle Derivierten Von Beschränkten Analytischen Functionen. Sitzungsber. Press. Acad. Viss. Berlin, Phys. Math. 4, 39-54 (1929)

14. Liu, TS, Wang, JF, Tang, XM: Schwarz lemma at the boundary of the unit ball in $\mathbb{C}^{n}$ and its applications. J. Geom. Anal. 25(3), 1890-1914 (2015)

15. Ren, GB, Wang, XP: Extremal functions of boundary Schwarz lemma. arXiv: 1502.02369

16. Unklebach, H: Über die Randverzerrung bei konformer Abbildung. Math. Z. 43, 739-742 (1938)

17. Herzig, A: Die Winkle derivierte und das Poisson-Stieltjes-Integral. Math. Z. 46, 129-156 (1940)

18. Osserman, R: A sharp Schwarz inequality on the boundary. Proc. Am. Math. Soc. 128, 3513-3517 (2000)

19. Sarason, D: Angular derivatives via Hilbert space. Complex Var. Elliptic Equ. 10, 1-10 (1988)

Submit your manuscript to a SpringerOpen ${ }^{\circ}$ journal and benefit from:

- Convenient online submission

Rigorous peer review

- Immediate publication on acceptance

- Open access: articles freely available online

- High visibility within the field

- Retaining the copyright to your article 